\title{
GAIA Level 3 Neonatal Death in a Non- viable Live Birth
}

National Cancer Institute

\section{Source}

National Cancer Institute. GAIA Level3 Neonatal Death in a Non-viable Live Birth. NCI

Thesaurus. Code C127971.

GAIA Level 3 Neonatal Death in a Non-viable Live Birth is defined by three criteria: first, the infant must be live born; second, the gestational age must be less than 5 months according to parent/family member/delivery attendant (a GA level of certainty of either level two or three); third, the death of the infant within the first 28 days of life must be documented. 